\title{
OCCURRENCE OF YEASTS, PSEUDOMONADS AND ENTERIC BACTERIA IN THE ORAL CAVITY OF PATIENTS UNDERGOING HEAD AND NECK RADIOTHERAPY
}

\author{
Elerson Gaetti-Jardim Júnior ${ }^{1}$, Francisco Isaak Nicolas Ciesielski ${ }^{1}$, Fátima Regina Nunes de Sousa ${ }^{1}$, Francisca \\ Nwaokorie $^{2}$, Christiane Marie Schweitzer ${ }^{3}$, Mario Júlio Avila-Campos ${ }^{4}$
}

${ }^{1}$ Departamento de Patologia e Propedêutica Clínica, Faculdade de Odontologia, Universidade Estadual Paulista, Araçatuba, SP, Brasil; ${ }^{2}$ Department of Medical Microbiology and Parasitology, College of Medicine, University of Lagos, Lagos, Nigeria; ${ }^{3}$ Departamento de Matemática, Faculdade de Engenharia, Universidade Estadual Paulista, Ilha Solteira, SP, Brasil; ${ }^{4}$ Departamento de Microbiologia, Instituto de Ciências Biomédicas, Universidade de São Paulo, São Paulo, SP, Brasil.

Submitted: June 11, 2010; Returned to authors for corrections: October 25, 2010; Approved: March 14, 2011.

\begin{abstract}
The aim of this study was to evaluate the occurrence of yeasts, pseudomonads and enteric bacteria in the oral cavity of patients undergoing radiotherapy (RT) for treatment of head and neck cancer. Fifty patients receiving RT were examined before, during and 30 days after RT. Saliva, mucosa, and biofilm samples were collected and microorganisms were detected by culture and polymerase chain reaction (PCR). The most prevalent yeasts in patients submitted to RT were Candida albicans, C. tropicalis, C. krusei, C. glabrata and C. parapsilosis. Citrobacter, Enterobacter, Enterococcus, Klebsiella, Proteus, and Pseudomonas were the most frequently cultivated bacteria. Before RT, targeted bacteria were cultivated from $22.2 \%$ of edentulous patients and $16.6 \%$ of dentate patients; 30 days after RT, these microorganisms were recovered from $77.8 \%$ edentulous and $46.8 \%$ dentate patients. By PCR, these microorganisms were detected from all edentulous patients, $78.1 \%$ of dentate patients. The presence of Gram-negative enteric roads and fungi was particularly frequent in patients presenting mucositis level III or IV. Modifications in the oral environment due to RT treatment seem to facilitate the colonization of oral cavity by members of family Enterobacteriaceae, genera Enterococcus and Candida.
\end{abstract}

Key words: Radiotherapy, Cancer, Enterobacteriaceae, Prevalence.

\section{INTRODUCTION}

The treatment of head and neck cancer (HNC) consists of surgery, radiotherapy (RT) or a combination of both. Chemotherapy has also been used as complement of this treatment (12). However, there are sufficient evidences showing that the use of radiotherapy in HNC treatment is detrimental due to several side effects, including: mucositis, xerostomia, radiation caries and osteoradionecrosis, which can complicate the possibility of intensification of cancer treatment $(2,23)$. In most cases, the occurrence and severity of these reactions depend on the radiation dose, volume of irradiated

*Corresponding Author. Mailing address: Laboratório de Microbiologia e Imunologia, Departamento de Patologia e Propedêutica Clínica, FOA, UNESP-Univ Estadual Paulista, R. Jose Bonifácio, 1193. 16015-050 Araçatuba, SP, Brazil.; Tel.: +55 18 3636-2797 Fax: +55 18 3636-4125.; E-mail: egaettij@ foa.unesp.br 
tissues, fractionation scheme, type of ionizing radiation, location of the irradiated area, patient's age, systemic conditions, concomitant treatment, oral hygiene, tobacco and alcohol consumption $(2,23)$.

In patients subjected to RT, the salivary gland dysfunction leads to decreased production of saliva, reduced clearance of carbohydrates and organic acids, decreased buffer capacity and tooth remineralization (21), in addition to changing the oral microbiota (14). Consequently, oral colonization and infection by opportunistic fungi and bacteria are commonly observed $(12,14)$. However, the qualitative and quantitative composition and alteration of the oral microbiota in HNC patients undergoing radiotherapy remains unclear. In addition, the role of opportunistic microorganisms in the pathogenesis of oral mucositis and other infections associated with side effects of $\mathrm{RT}$ is not conclusive.

The involvement of microorganisms such as fungi and enteric species in the pathogenesis of oral infections in patients with severe immune deficiency has been widely discussed (23). These enteric microorganisms frequently act as reservoirs for antimicrobial resistance genes, which spread to other microbial populations (8).

The use of nystatin suspension in the candidosis treatment is a standard practice in patients subjected to $\mathrm{RT}$ in some Brazilian radiotherapy centers, but this protocol is also very dependent upon patients' compliance. Unfortunately, there is no clinical or microbiological evidence to show that this therapeutic scheme is effective in patients with low socioeconomic status and poor oral hygiene.

Moreover, since most of Brazilian patients undergoing RT for treatment of HNC present low socioeconomic conditions and insufficient oral hygiene standards, as well as wear complete dentures, it is possible that these factors contribute significantly to the establishment of opportunistic microorganisms, exacerbating the side effects of RT and compromising the intensification of cancer treatment. Thus, the aim of this study was to evaluate the occurrence of yeasts, pseudomonads and enteric bacteria in oral microbiota of patients with head and neck cancer undergoing RT.

\section{MATERIAL AND METHODS}

\section{Study Population and Clinical Examinations}

A total of 50 patients (38 males and 12 females) seen at the Center for Radiotherapy of São José do Rio Preto, São Paulo, Brazil, aged 16-80 years (mean age 59.5 years), with histopathological diagnosis of malignant disease were included in the study. Forty-four patients presented squamous cell carcinoma, four patients harbored acinar cell carcinoma, one patient presented basal cell squamous carcinoma and another one presented malignant undifferentiated neoplasm.

Most of patients were illiterate or presented elementary formal education (84\%), as well as tobacco (90\%) and/or alcohol $(74 \%)$ consumers. Eighteen patients (36\%) were totally edentulous and wore complete dentures and 32 patients $(64 \%)$ had at least six teeth (mean $12.4 \pm 5.7$ ). Fourteen of these patients had periodontitis and 18 presented gingivitis. A fullmouth periodontal examination was performed. Clinical assessments included probing depth, recession, bleeding on probing (yes/no), and determining the plaque index (presence/absence). The clinical attachment loss was calculated by adding the probing depth and recession measurements. Periodontal measurements were carried out at six sites per tooth (mesio-buccal, buccal, disto-buccal, mesio-lingual, lingual and disto-lingual) for all teeth. The medical staff at the Center for Radiotherapy provided data on systemic health and medication taken by the patients.

Patients were instructed by the medical staff at the Center for Radiotherapy to seek dental treatment prior to RT. However, only $38 \%$ of the patients sought dental treatment which consisted of dental extractions, periodontal treatment and restorative procedures. In addition, all patients were instructed to use $5 \mathrm{ml}$ of an oral suspension containing 100,000 $\mathrm{U} / \mathrm{ml}$ aqueous nystatin three times a day (Micostatin ${ }^{\circledR}$, Bristol- 
Myers Squibb, São Paulo, SP, Brazil) in order to prevent oral candidosis during RT.

Clinical examinations were performed with the patients before dental treatment and RT (stage 1), immediately after completion of RT (stage 2), and 30 days after RT (stage 3). During these visits to the Center for Radiotherapy, the presence and severity of mucositis in patients was assessed according to criteria provided by the National Center Institute (22) and xerostomia was evaluated using clinical criteria and by a specific form (9).

The patients were submitted to RT for the treatment of head and neck cancer, and the mean radiation dose received varied from 4320 to $7020 \mathrm{cGy}$ (mean dose 6233 cGy \pm 954.5 cGy) and the fractioning dose was 180 cGy. Radiotherapy was carried out using a linear accelerator.

Patients exhibiting uncontrolled cardiovascular disease, pulmonary disease, diabetes mellitus, renal or hepatic disease and additional systemic diseases were excluded, as well as patients who needed antibiotic prophylaxis, steroids and antibiotic therapy in the last 6 months prior to RT. All patients gave written informed consent to be included in this study, which was approved by the Institutional Review Board of School of Dentistry of Araçatuba-UNESP (No. 01559/2007).

\section{Clinical samples, microbial isolation and identification}

Before clinical examination, whole resting saliva, oral mucosa (including dorsum of the tongue), supragengival and subgingival biofilms were sampled. Saliva was collected using a draining method; patients were placed in a quiet room and asked not to drink, eat or clean their mouths 1 hour before saliva collection. They were further instructed not to swallow any saliva during the collection period.

The oral mucosa was sampled using a sterile swab and transferred to a solution consisting of $300 \mu \mathrm{L}$ of $\mathrm{PBS}$ (phosphate buffered solution) and $300 \mu \mathrm{L}$ of ultra pure water. Supragingival biofilm was sampled by scaling. Subgingival samples of the three most diseased periodontal sites were collected using sterilized paper points introduced into the apical region of the periodontal pocket or inflamed gingival crevice and allowed to remain for 60 seconds. The paper points were pooled and transferred to $300 \mu \mathrm{L}$ of PBS and $300 \mu \mathrm{L}$ of ultra pure water. Clinical samples were processed within 2 hours.

Specimens were inoculated in peptone water and ethyl violet azide broth (EVA broth, Difco) and incubated 3-7 days at room temperature. Subsequently, from microbial growth observed in peptone water, aliquots of $0.1 \mathrm{ml}$ were transferred to Eosin Methylene Blue agar, MacConkey agar, Brilliant Green agar and Brain Heart Infusion agar supplemented with defibrinated horse blood, incubated at $37^{\circ} \mathrm{C}$, for $24-48 \mathrm{~h}$. Samples were also inoculated onto Sabouraud Dextrose agar with $100 \mu \mathrm{g} / \mathrm{ml}$ of chloramphenicol and incubated at room temperature, for 3-7 days, for yeasts isolation. From tubes containing EVA broth, aliquots of $0.1 \mathrm{~mL}$ were transferred to Bile Esculin agar and incubated in aerobiosis for 48 h. at $37^{\circ} \mathrm{C}$, in order to isolate enteric bacteria and pseudomonads (7).

The yeast identification was performed by carbon and nitrogen assimilation tests, fermentation of carbohydrates, germ tube formation (at $37^{\circ} \mathrm{C}$ and at $39^{\circ} \mathrm{C}$ ), colonial morphology on CHROMagar Candida (MastDiagnostica, Paris, France), and growth at $37^{\circ} \mathrm{C}$ and $42^{\circ} \mathrm{C}$. In the carbohydrate assimilation tests, the inoculum cultivated overnight in yeast peptone dextrose broth, was adjusted to 5 McFarland standard, centrifuged for $5 \mathrm{~min}$. at 2000.g and the pellets were 3 times washed and added to $1 \mathrm{~mL}$ of sterile Yeast Nitrogen Base (YNB, Difco) and $20 \mathrm{~mL}$ of bacteriological agar (Bacto Agar, Difco), mixed and plated in $15 \mathrm{~cm}$ Petri dishes. After solidification of the medium, discs with $2 \%$ carbohydrates (maltose, trehalose, xylose, galactose, lactose, sucrose and glucose) were plated and incubated for $96 \mathrm{~h}$. at $30^{\circ} \mathrm{C}$. The presence of microbial growth around paper discs containing carbohydrates was registered daily. The Candida isolates with inconclusive identification to species level were submitted to DNA extraction and amplification following the 
methodology previously described, using PCR with specific primer pairs (13).

Bacterial species were identified using the following methods: colonial characteristics, Gram-staining, growth at $10 \%$ sodium chloride, production of gas from glucose, and biochemical tests using the API-20E commercial kit (BioMérieux SA, Marcy-l'Etoile, France). Some bacterial isolates were identified using the BBL Crystal Enteric/Nonfermenter system (Becton Dickinson Microbiology Systems, Cockeysville, MD), following recommendations of the manufacturer $(1,15)$.

\section{PCR assay}

The DNA of all clinical samples transported in sterile ultra pure water was extracted using QIAamp DNA Mini Kit (Qiagen, Hilden, Germany), following the manufacturer's instructions. Concentrations of bacterial DNA were determined with a spectrophotometer (Beckman Instruments, Richmond, Washington, USA) at $\mathrm{A}_{260} \mathrm{~nm}$. The presence of Enterobacteriaceae Enterococcus spp., E. faecalis, E. faecium, Pseudomonas spp. and P. aeruginosa was detected by PCR using specific primer pairs (6). DNA from $P$. aeruginosa ATCC 10145, P. putida ATCC 49128, E. coli ATCC 35218 and ATCC 25922, Acinetobacter haemolyticus ATCC 19002, E. faecium ATCC 35667, and E. faecalis ATCC 29212 was used as positive control.

PCR amplification was performed in volumes of $25 \mu \mathrm{L}$ containing $1 \mathrm{X}$ PCR/ $\mathrm{Mg}^{++}$buffer (Boehring Mannheim, Indianapolis, IN, USA), $0.2 \mathrm{mM}$ each of dNTP (Pharmacia Biotech, Piscataway, NJ, USA), 0.5 U Taq DNA polymerase (Invitrogen, São Paulo, SP, Brazil), $0.4 \mu \mathrm{M}$ of each primer pair (Invitrogen) and $10 \mathrm{ng}$ of template. Amplification was performed in a DNA Thermal Cycler (Perkin Elmer, GeneAmp PCR System 9700, Norwalk, CT, USA) programmed for $94^{\circ} \mathrm{C}$ for 5 minutes, followed by 35 cycles at $94^{\circ} \mathrm{C}$ for 30 seconds; annealing temperature specific for each primer pair, ranging between 30 seconds and 1 minute; $72^{\circ} \mathrm{C}$ for 1 minute, then $72^{\circ} \mathrm{C}$ for 5 minutes to allow completion of DNA extension. Amplification products were compared by electrophoresis in a solution of $1 \%$ agarose gel in $1 \mathrm{X}$ TBE buffer stained with ethidium bromide $(0.5 \mathrm{mg} / \mathrm{mL})$.

\section{Statistical analysis}

Statistical analyses were performed using the software Statistical Package for the Social Sciences (SPSS Incs v.13, Chicago, IL, USA). The frequency of detection of the microorganisms was computed for each subject. Significant differences between patients were tested using the MannWhitney test, Chi-square test or Fisher's exact test. Differences of $p<0.05$ was considered statistically significant.

\section{RESULTS}

Before radiation treatment, patients had no clinical evidence or complaint of mucositis, dermatitis, candidosis, xerostomia, or dysgeusia. However, immediately after the end of radiotherapy, these side effects could be observed in $90 \%$, $86 \%, 54 \%, 94 \%$ and $88 \%$ of patients, respectively. These percentages were reduced to $80 \%, 76 \%, 30 \%, 62 \%$ and $76 \%$, 30 days after completion of treatment. Immediately after RT, only five patients showed no clinical signs of mucositis, eight presented mucositis level I, nine presented mucositis level II, twenty-one presented mucositis level III and seven individuals presented extensive ulcerations (mucositis level IV). The occurrence of mucositis was associated with development of xerostomia (Chi-square test, $p=0.021$ ).

In relation to gender, there was no correlation with the occurrence of mucositis, dermatitis (Fisher's exact test, p = 0.578), dysgeusia (Fisher's exact test, $p=1.0$ ) or xerostomia (Fisher's exact test, $p=0.568$ ). The presence of complete dentures or dental conditions (i.e., presence or absence of cavitated caries) did not influence the occurrence of mucositis (Chi-square test, $\mathrm{p}=0.139$ ), dermatitis (Chi-square test, $\mathrm{p}=$ 0.822), dysgeusia (Chi-square test, $\mathrm{p}=0.792$ ) or xerostomia 
(Chi-square test, $\mathrm{p}=0.862$ ). The periodontal status did not influence the occurrence of mucositis (Chi-square test, $\mathrm{p}=0.779$ ), dermatitis (Chi-square test, $\mathrm{p}=0.940)$, xerostomia (Chi-square test, $\mathrm{p}=0.382)$ or dysgeusia (Chi-square test, $\mathrm{p}=0.205)$.

It was difficult to evaluate the influence of the radiation dose on the occurrence and severity of mucositis, as well as other side effects of RT, because most of the patients received $7020 \mathrm{cGy}$ (66\%). The number of patients who received 5040 cGy $(20 \%)$, 6660 cGy (10\%) and 4320 cGy (4\%) was too small to allow statistically reliable conclusions. There was no significant correlation between RT regimens described above and the incidence of mucositis, dermatitis (Fisher's exact test, $p=0.398$ ), dysgeusia (Fisher's exact test, $p=0.392$ ) or xerostomia (Fisher's exact test, $p=0.191)$. Neither was there a correlation between the level of formal education, alcohol and tobacco consumption and degree of oral side effects of RT.

Initially, before radiotherapy, enteric microorganisms were cultivated from samples of oral mucosa from four edentulous patients $(22.5 \%)$ and from subgingival biofilms of three dentate patients (9.4\%). Isolates were identified as E. faecalis in six samples from edentulous or dentate patients, and Citrobacter freundii was identified in a sample from an edentulous patient. Additionally, 30 days after RT, enteric bacteria and pseudomonads were cultivated from $77.8 \%$ of the edentulous patients and from $46.9 \%$ of the dentate patients. The identification of cultivated bacteria is presented in Table 1, and the most frequently detected genera were Citrobacter, Enterobacter, Enterococcus, Klebsiella, Morganella, Proteus, and Pseudomonas. Moreover, edentulous patients presented higher frequency of oral colonization by enteric microorganisms $(p=0.038)$ and radiotherapy was associated with significant increase in the occurrence of these microorganisms $(p<0.001)$.

These data were confirmed by PCR (Table 2). With regard to pseudomonads, when the data obtained from cultures are considered, radiotherapy seems to increase the frequency of colonization of the oral cavity by these microorganisms. However, when the PCR data is considered, this is no longer statistically significant. As evidenced by PCR, before RT, targeted bacteria were detected in $55.6 \%$ of the edentulous patients, and in $43.8 \%$ of the dentate patients, while 30 days after RT, these values increased to $100 \%$ and $78.1 \%$, respectively. The occurrence of enteric microorganisms was not associated with gingivitis or periodontitis, but they were observed in higher frequency among tobacco consumers $(\mathrm{p}=0.031)$, and patients presenting level III or IV mucositis $(\mathrm{p}=0.042)$.

Table 1. Targeted bacteria cultivated from patients before RT and 30d. after RT.

\begin{tabular}{ccc}
\hline Microorganism & $\mathbf{E P}^{1} \mathbf{N}(\%)$ & $\mathbf{D P}^{2} \mathbf{N}(\%)$ \\
\hline before radiotherapy & $1(5.6)$ & $0(0.0)$ \\
Citrobacter freundii & $3(16.7)$ & $3(16.6)$ \\
Enterococcus faecalis & $4(22.2)$ & $3(16.6)$ \\
Total & & \\
30 d after radiotherapy & $2(11.1)$ & $5(15.6)$ \\
Citrobacter freundii & $3(16.7)$ & $4(12.5)$ \\
Enterobacter cloacae & $4(22.2)$ & $2(6.3)$ \\
E. intermedius & $4(22.2)$ & $2(6.3)$ \\
E. sakazakii & $2(11.1)$ & $0(0.0)$ \\
Enterococcus sp. & $6(33.3)$ & $6(18.8)$ \\
E. faecalis & $1(5.6)$ & $1(3.1)$ \\
Escherichia coli & $6(33.3)$ & $3(9.4)$ \\
Klebsiella oxytoca & $2(11.1)$ & $2(6.3)$ \\
Morganella morganii & $1(5.6)$ & $1(3.1)$ \\
Pantoea agglomerans & $3(16.7)$ & $3(9.4)$ \\
Proteus mirabilis & $1(5.6)$ & $0(0.0)$ \\
Providencia alcalifaciens & $2(11.1)$ & $0(0.0)$ \\
Pseudomonas sp. & $4(22.2)$ & $2(6.3)$ \\
P. aeruginosa & $14(77.8)$ & $15(46.9)$ \\
Total $*$ & $*$ Some patients &
\end{tabular}

${ }^{T}$ Edentuous patients $\mathrm{N}=18 ;{ }^{2}$ Dentate patients $\mathrm{N}=32$. *Some patients harbored more than a single species of targeted microorganism. 
Table 2. Targeted bacteria from irradiated patients before and 30 days after RT. Results obtained by PCR.

\begin{tabular}{|c|c|c|}
\hline \multirow{2}{*}{ Microorganism } & \multicolumn{2}{|c|}{ Patients } \\
\hline & $\mathbf{E P}^{1} \mathbf{N}(\%)$ & $D^{2} \mathbf{N}(\%)$ \\
\hline \multicolumn{3}{|l|}{ before radiotherapy } \\
\hline Enterococcus sp. & $5(27.8)$ & $6(18.8)$ \\
\hline E. faecalis & $3(16.7)$ & $4(12.5)$ \\
\hline E. faecium & $1(5.6)$ & $2(6.3)$ \\
\hline Enterobacteriaceae & $5(27.8)$ & $7(21.9)$ \\
\hline Pseudomonas sp. & $4(22.2)$ & $3(9.4)$ \\
\hline$P$. aeruginosa & $2(12.5)$ & $1(3.1)$ \\
\hline Total* & $10(55.6)$ & $14(43.8)$ \\
\hline \multicolumn{3}{|l|}{$30 \mathrm{~d}$ after radiotherapy } \\
\hline Enterococcus sp. & $11(61.1)$ & $11(34.4)$ \\
\hline E. faecalis & $8(44.4)$ & $11(34.4)$ \\
\hline E. faecium & $2(11.1)$ & $2(6.3)$ \\
\hline Enterobacteriaceae & $12(66.7)$ & $19(59.4)$ \\
\hline Pseudomonas sp. & $5(27.8)$ & $5(15.6)$ \\
\hline$P$. aeruginosa & $4(22.2)$ & $5(15.6)$ \\
\hline Total ${ }^{*}$ & $18(100.0)$ & $25(78.1)$ \\
\hline
\end{tabular}

Fifteen patients $(30 \%)$ were initially colonized by Candida spp., increasing to $78 \%$ soon after RT and $84 \% 30$ days after RT, and this increase was not directly related to the dental or periodontal status (Table 3). Thirty days after radiotherapy, yeasts were recovered from all edentulous subjects and from $75 \%$ of the dentate patients. Fungi isolates were identified as Candida albicans, C. tropicalis, C. krusei, C. glabrata or C. parapsilosis. The presence of $C$. albicans concomitantly with non-albicans Candida species was particularly common after RT, and the occurrence of Candida spp. was increased by RT $(\mathrm{p}<0.001)$.

Table 3. Candida species isolated from oncologic patients at different moments of the study.

\begin{tabular}{|c|c|c|c|c|c|c|}
\hline \multirow{2}{*}{ Microorganism } & \multicolumn{2}{|c|}{ Baseline $^{1}$ N (\%) } & \multicolumn{2}{|c|}{ IAR $^{2}$ N (\%) } & \multicolumn{2}{|c|}{30 d RT $^{3}$ N (\%) } \\
\hline & $E P^{4}$ & $\mathrm{DP}^{5}$ & EP & DP & EP & DP \\
\hline C. albicans & $9(50.0)$ & $6(18.8)$ & $18(100.0)$ & $21(65.6)$ & $18(100.0)$ & $20(62.5)$ \\
\hline C. tropicalis & $1(5.6)$ & $1(3.1)$ & $5(27.8)$ & $1(3.1)$ & $5(27.8)$ & $4(12.5)$ \\
\hline C. krusei & $1(5.6)$ & $2(6.3)$ & $2(11.1)$ & $2(6.3)$ & $4(22.2)$ & $2(6.3)$ \\
\hline C. glabrata & $0(0.0)$ & $0(0.0)$ & $2(11.1)$ & $0(0.0)$ & $3(16.7)$ & $2(6.3)$ \\
\hline C. parapsilosis & $0(0.0)$ & $0(0.0)$ & $1(5.6)$ & $0(0.0)$ & $3(16.7)$ & $1(3.1)$ \\
\hline Total & $9(50.0)$ & $6(18.8)$ & $18(100.0)$ & $21(65.6)$ & $18(100.0)$ & $24(75.0)$ \\
\hline Mean $\pm \mathrm{SD}^{6}$ & $305.8 \pm 204.5$ & $23 \pm 14.5$ & $1117 \pm 903.6$ & $77.2 \pm 52.3$ & $6305.7 \pm 2041.9$ & $468 \pm 209$ \\
\hline
\end{tabular}

${ }^{1}$ Baseline: immediately before RT; ${ }^{2}$ IAR: immediately after completion of RT; ${ }^{3} 30$ days after RT; ${ }^{4}$ edentulous patients; ${ }^{5}$ dentate patients; ${ }^{6}$ mean CFU counts \pm standard deviations in positive samples.

\section{DISCUSSION}

The risk of mucositis has been a major concern for patients who receive RT for treatment of head and neck cancer $(12,18)$. Susceptibility to mucositis is related to qualitative and quantitative changes in saliva, oral microbiota and immunological status (14). Consequently, preventive measures before, during and after radiotherapy are necessary and should include instructions regarding diet, oral hygiene and the use of chemical compounds to prevent microbial accumulation on dental or prosthetic surfaces.

The results of this study indicate that radiotherapy increases the presence of enteric bacteria and Candida species during the period immediately after RT in accordance with 
other studies $(1,20)$ and several factors may contribute to the oral colonization by these microbial species, such as radiationinduced hyposalivation, smoking, alcohol abuse and presence of prosthesis (5). Moreover, most of the patients were tobacco and alcohol consumers and only six patients not considered themselves alcohol-dependent. In addition, 30\% of patients wearing complete or partial dentures showed bad conditions of hygiene and maintenance of prosthesis and it could facilitate the increase of such microorganisms (3)

Many factors support the establishment of enteric microorganisms in the oral cavity of irradiated patients, including poor oral and corporal hygiene standards, which are commonly observed in most patients with head and neck cancer (10), and in patients with complete and partial dentures where these hygiene conditions apparently exacerbated the discomfort produced by xerostomia and mucositis, impairing their precarious efficiency in oral hygiene procedures.

The transmission of enteric cocci and rods to the oral cavity of patients undergoing RT for treatment of head and neck cancer by means of drinking-water or food contaminated by sewage is a real concern, but this phenomenon was not previously studied with oncologic patients. Sixteen percent of the patients were farmers and the water treatment in rural areas of Brazil is still rare, including the treatment of domestic sewage, which is practically non-existent. In addition, other $40 \%$ of patients lived in suburbs, where water sources contaminated by domestic sewage are not uncommon.

Despite the high frequency of enteric bacteria and pseudomonads, their role in the pathogenesis of mucositis remains unclear. Gram-negative bacteria may intensify the inflammatory process as well as exacerbate ulcer formation by the release of proteases and endotoxins (8), which interact with host's cells to induce the production and secretion of proinflammatory cytokines (17).

Although the colonization of the oral cavity by these microorganisms may be transient (14), the results of this investigation suggest that these microorganisms are likely to remain in the oral environment for weeks or longer after RT. Since the number of detected species and their prevalence increased during and shortly after RT, there is no evidence to suggest that their presence in the oral cavity is transient or significantly reduced in the period subsequent to that indicated in this study.

Thus, the higher occurrence of yeasts, enteric cocci and rods observed in this study may be due to the evaluated population that showed several predisposing factors for the occurrence of mycoses and opportunistic infections. In addition, xerostomia and mucositis may interfere with food intake and may exacerbate the chronic malnutrition that is common among the poorest people as the patients evaluated here. Malnutrition may predispose the patient to opportunistic infections, which is often observed in the Brazilian countryside.

The incidence of fungal infections has been reported in $40 \%$ of the patients with malignancies (4), or in those undergoing RT as also observed in this study. Candida albicans, C. tropicalis, C. parapsilosis $(4,12)$, C. krusei, C. glabrata (19), C. dubliniensis or C. guilliermondii (4) are commonly observed in infections in oncologic or irradiated patients.

In the present study, most of patients were middle-aged male adults, who were chronic tobacco and alcohol consumers and had advanced malignant tumors. It is known that the use of tobacco, alcohol and oral prostheses may increase colonization by opportunistic pathogens, particularly fungi $(16,18)$. Moreover, radiotherapy seems to increase the frequency and intensity of oral colonization by Candida spp.

The results of this study confirm the increased number of non-C. albicans species in the microbiota of irradiated patients, but this phenomenon was not at the expense of a reduction of C. albicans occurrence, as described in the literature $(4,19)$, showing that several Candida species may increase their occurrence in the oncologic patients. In a previous study (12), although $C$. albicans was the most frequently detected yeast 
before RT, this microorganism was not cultivated at the end of RT, what can reflect the extensive use of miconazole, nystatin and/or ketoconazole in the treatment of oral candidosis as described by the authors, who used only saliva for yeasts cultivation and in the present investigation supragingival and subgingival biofilms were also collected in order to provide a more extensive evaluation of the presence of the Candida species in the oral cavity.

The use of topical nystatin to reduce candidosis has become a standard practice in patients submitted to RT in some Brazilian radiotherapy centers, but this protocol is also very compliance-dependent. Although intraoral and esophageal candidosis may be treated effectively with nystatin (11), the high incidence of candidosis and the frequency of the Candida carriage reported herein suggest that this topical antifungal agent fails to prevent the colonization or intraoral dissemination of yeasts in patients submitted to RT. In order to reduce Candida spp. populations as well as the occurrence of candidosis, oral administration of fluconazole may be prescribed (11).

\section{ACKNOWLEDGEMENTS}

This study was partially supported by grants of Fundação de Amparo à Pesquisa do Estado de São Paulo (FAPESP, Proc. 2007/54851-0).

\section{REFERENCES}

1. Almstahl, A.; Wikström, M.; Fagerberg-Mohlin, B. (2008). Microflora in oral ecosystems in subjects with radiation induced hyposalivation. Oral Dis. 14, 541-549.

2. Bonan, P. R. F.; Lopes, M. A.; Pires, F. R.; Almeida, O. P. (2006) Dental management of low socioeconomic level patients before radiotherapy of the head and neck with special emphasis on the prevention of osteoradionecrosis. Braz. Dent. J. 17, 336-342.

3. Daniluk, T.; Fiedoruk, K.; Sciepuk, M.; Zaremba, M. L.; Rozkiewicz, D.; Cylwik-Rokicka, D.; Tokajuk, G.; Kedra, B. A.; Anielska, I.; Stokowska, W.; Górska, M.; Kedra, B. R. (2006). Aerobic bacteria in the oral cavity of patients with removable dentures. Adv. Med. Sci. 51 (Suppl 1), 86-90.
4. Davies, A. N.; Railsford, S.; Broadley, K.; Beighton, D. (2002). Oral yeast carriage in patients with advanced cancer. Oral Microbiol. Immunol. 17, 79-84.

5. Epstein, J. B.; Frelich, M. M.; Le, N. D. (1993). Risk factors for oropharyngeal candidiasis in patients who receive radiation therapy for malignant conditions of the head and neck. Oral Surg. Oral Med. Oral Pathol. Oral Radiol. Endod. 76, 169-174.

6. Gaetti-Jardim Jr, E.; Fardin, A. C.; Gaetti-Jardim, E. C.; Castro, A. L.; Schweitzer, C. M. (2010). Microbiota associated with chronic osteomyelitis of the jaws. Braz. J. Microbiol. 41, 1056-1064.

7. Gaetti-Jardim Jr, E.; Nakano, V.; Wahasugui, T. C.; Cabral, F. C.; Gamba, R.; Avila-Campos, M. J. (2008). Occurrence of yeasts, enterococci and other enteric bacteria in subgingival biofilm of HIVpositive patients with chronic gingivitis and necrotizing periodontitis. Braz. J. Microbiol. 39, 257-261.

8. Gonçalves, M. O.; Coutinho-Filho, W. P.; Pimenta, F. P.; Pereira, G. A.; Pereira, J. A. A.; Mattos-Guaraldi, A. L.; Hirata Jr, R. (2007). Periodontal disease as reservoir for multi-resistant and hydrolytic enterobacterial species. Lett. Appl. Microbiol 44, 488-494.

9. Guggenheimer, J.; Moore, P. A. (2003). Xerostomia. Etiology, recognition and treatment. J. Amer. Dent. Assoc. 134, 61-69.

10. Hasturk, H.; Nunn, M.; Warbington, M.; Van Dyke, T. E. (2004). Efficacy of a fluoridated hydrogen peroxide-based mouthrinse for the treatment of gingivitis: a randomized clinical trial. J. Periodontol. 75, 5765.

11. Ilgenli, T.; Ören, H.; Uysal, K. (2001). The acute effects of chemotherapy upon the oral cavity: Prevention and management. Turk. $J$. Cancer 31, 93-105.

12. Jham, B. C.; França, E. C.; Oliveira, R. R.; Santos, V. R.; Kowalski, L. P.; Freire, A. R. S. (2007). Candida oral colonization and infection in Brazilian patients undergoing head and neck radiotherapy: a pilot study. Oral Surg. Oral Med. Oral Pathol. Oral Radiol. Endod. 103, 355-358.

13. Khan, Z. U.; Mustafa, A. S. (2001). Detection of Candida species by polymerase chain reaction (PCR) in blood samples of experimentally infected mice and patients with suspected candidemia. Microbiol. Res. $156,95-102$

14. Leung, W. K.; Jin, L. J.; Yam, W. C.; Samaranayake, L. P. (2001). Oral colonization of aerobic and facultatively anaerobic gram-negative rods and cocci in irradiated, dentate, xerostomic individuals. Oral Microbiol. Immunol. 16, 1-9.

15. Marinho, S. A.; Teixeira, A. B.; Santos, O. S.; Cazanova, R. F.; Ferreira, C. A. S.; Cherubini, K.; Oliveira, S. D. (2010). Identification of Candida spp. by phenotypic tests and PCR. Braz. J. Microbiol. 41, 286-294.

16. Mizugai, H.; Isogai, E.; Chiba, I. (2007). Effect of denture wearing on occurrence of Candida species in the oral cavity. J. Appl. Res. 7, 250254.

17. Napeñas, J. J.; Brennan, M. T.; Bahrani-Mougeot, F. K.; Fox, P. C.; 
Lockhart, P. B. (2007). Relationship between mucositis and changes in oral microflora during cancer chemotherapy. Oral Surg. Oral Med. Oral Pathol. Oral Radiol. Endod. 103, 48-59.

18. Ramirez-Amador, V.; Silverman Jr, S.; Mayer, P.; Tyler, M.; Quivey, J. (1997). Candidal colonization and oral candidiasis in patients undergoing oral and pharyngeal radiation therapy. Oral Surg. Oral Med. Oral Pathol. Oral Radiol. Endod. 84, 149-153.

19. Redding, S. W.; Zellars, R. C.; Kirkpatrick, W. R.; McAtee, R. K.; Caceres, M. A.; Fothergill A. W.; Lopez-Ribot, J. L.; Bailey, C. W.; Rinaldi, M. G.; Paterson, T. F. (1999). Epidemiology of oropharyngeal Candida colonization and infection in patients receiving radiation for head and neck cancer. J. Clin. Microbiol. 37, 3896-3900.
20. Samaranayake, L. P.; Robertson, A. G.; MacFarlane, T. W.; Hunter, I. P.; MacFarlane, G.; Soutar, D. S.; Ferguson, M. M. (1988). The effect of chlorhexidine and benzydamine mouthwashes on mucositis induced by therapeutic irradiation. Clin. Radiol. 39, 291-294.

21. Shiboski, C. H.; Hodgson, T. A.; Ship, J. A.; Schiodt, M. (2007). Management of salivary hypofunction during and after radiotherapy. Oral Surg. Oral Med. Oral Pathol. Oral Radiol. Endod. 103, 61-66.

22. Trotti, A. (2000). Toxicity in head and neck cancer: a review of trends and issues. Int. J. Rad. Oncol. Biol. Phys. 47, 1-12.

23. Vissink, A.; Jansma, J.; Spijkervet, F. K. L.; Burlage, F. R.; Coppes, R. P. (2003). Oral sequelae of head and neck radiotherapy. Crit. Rev. Oral. Biol. Med. 14, 199-212. 